\title{
Efficacy and Safety of Resveratrol Supplements on Blood Lipid and Blood Glucose Control in Patients with Type 2 Diabetes: A Systematic Review and Meta-Analysis of Randomized Controlled Trials
}

\author{
Tianqing Zhang, ${ }^{1}$ Qi He $\mathbb{D}^{2},{ }^{2}$ Yao Liu, ${ }^{1}$ Zhenrong Chen, ${ }^{1}$ and Hengjing Hu $\mathbb{C}^{1,3}$ \\ ${ }^{1}$ The First Affiliated Hospital, Department of Cardiovascular Medicine, Hengyang Medical School, University of South China, \\ Hengyang, Hunan Province, China \\ ${ }^{2}$ People's Hospital of Ningxiang City, Ningxiang City, Hunan Province, China \\ ${ }^{3}$ Institute of Cardiovascular Disease and Key Lab for Arteriosclerology of Hunan Province, University of South China, Hengyang, \\ Hunan, China \\ Correspondence should be addressed to Hengjing Hu; bestmanhhj123@163.com
}

Received 3 June 2021; Accepted 19 July 2021; Published 25 August 2021

Academic Editor: Thanasekaran Jayakumar

Copyright (c) 2021 Tianqing Zhang et al. This is an open access article distributed under the Creative Commons Attribution License, which permits unrestricted use, distribution, and reproduction in any medium, provided the original work is properly cited.

\begin{abstract}
Background. Diabetes is a major public health concern. Resveratrol has shown great beneficial effects on hyperglycemia and insulin resistance and as an antioxidant. Methods. We searched the Chinese and English databases (such as CNKI, PubMed, and Embase) and extracted data from randomized controlled trials (RCTs). Then, RevMan 5.3 was used for bias risk assessment and meta-analysis. The primary outcome indicators include insulin-resistance-related indicators and blood-lipid-related indicators. This systematic review and meta-analysis was registered in PROSPERO (CRD42018089521). Results. Fifteen RCTs involving 896 patients were included. For insulin-resistance-related indicators, the summary results showed that, compared with the control group, homeostasis model assessment for insulin resistance (HOMA-IR) in the resveratrol group is lower (WMD: -0.99 ; $95 \%$ CI $-1.61,-0.38 ; P=0.002$ ). For blood-lipid-related indicators, the total cholesterol (TC) and triglyceride (TG) in the resveratrol group is of no statistical significance (for TC, WMD: -7.11 ; 95\% CI $-16.28,2.06$; $P=0.13$; for TG, WMD: -2.15 ; $95 \%$ CI -5.52 , $1.22 ; P=0.21)$. For adverse events, the summary results showed that there was no statistical difference in the incidence of adverse events between the resveratrol and control groups (WMD: 2; 95\% CI 0.44, 9.03; $P=0.37$ ). Conclusion. Based on the current evidence, resveratrol may improve insulin resistance, lower fasting blood glucose and insulin levels, and improve oxidative stress in patients with type 2 diabetes mellitus.
\end{abstract}

\section{Introduction}

Diabetes is a serious metabolic disease that affects about $5 \%$ of the world's people. Epidemiological data show that the number of people with diabetes is expected to increase dramatically to 592 million by 2035 [1]. 12\% of global health expenditure is spent annually on diabetes and its complications [2]. Diabetes is divided into different types: type 1 and type 2 diabetes account for more than $90 \%$ of all cases. Metabolic abnormalities and serious complications caused by type 2 diabetes have profound effects on the life and quality of life of patients, such as microvascular (retinopathy, nephropathy), large blood vessels and peripheral vascular disease [3, 4], and increased risk of cancer [5, 6]. Type 2 diabetes mellitus (T2DM) is characterized by insulin resistance and hyperglycemia [7]. The treatment drugs for T2DM include insulin, alpha glucosidase inhibitors, dipeptidyl peptidase 4 inhibitors, incretin analogues, biguanides, insulin secretagogues, insulin sensitizers, and intestinal lipase inhibitors $[8,9]$. However, the currently used therapies are accompanied by side effects, such as hypoglycemia, gastrointestinal problems, and weight gain [8]. Therefore, new drugs and natural compounds are constantly being tested to better prevent and treat diabetes [10]. 
In the alternative treatment strategy for diabetes treatment, resveratrol, a naturally occurring polyphenolic compound, mainly derived from the rhizome of the main natural source of Polygonum cuspidatum. Studies have shown that resveratrol has shown great beneficial effects on hyperglycemia, insulin resistance, and antioxidant [11, 12]. Clinical trials have shown that resveratrol has potential benefits for patients with T2DM, and relevant systematic reviews and reviews have also made relevant comments. However, some results contradict the evidence for the beneficial effects of resveratrol in the treatment of T2DM $[13,14]$. This may be due to the limitation of sample size and treatment duration masking clear changes in clinical practice [12-14]. Meanwhile, the most recent meta-analysis search deadline was in June 2017, and a large number of RCTs appeared in the following period [15-20]. Therefore, we conduct a new systematic review and meta-analysis on this topic to evaluate the effects of resveratrol supplements on blood sugar, blood lipids, oxidative stress, safety, and other aspects of T2DM.

\section{Materials and Methods}

2.1. Protocol. This systematic review and meta-analysis were conducted strictly in accordance with the protocol (CRD42018089521) and PRISMA 2020 guidelines (see Supplementary Materials).

\subsection{Inclusion and Exclusion Criteria}

2.2.1. Participants. Participants are patients with T2DM diagnosed through recognized standards, regardless of age, gender, and nationality. Records need to mention clear diagnostic criteria for RA, with a balanced baseline and comparability.

2.2.2. Intervention. The intervention in experiments group was resveratrol supplements with no limits on the type, dose, frequency, and so on. The intervention in control group was western medicine, blanks, or placebo.

2.2.3. Outcomes. The primary outcomes are as follows: homeostasis model assessment for insulin resistance (HOMA-IR), total cholesterol (TC), triglyceride (TG). The Secondary outcomes are as follows: HbA1c, low density lipoprotein cholesterol (LDL-C), high density lipoprotein cholesterol (HDL-C), fasting glucose, fasting insulin, and MDA.

2.2.4. Study Type. This study is a randomized controlled trial (RCT), with no limits on the manner by which randomization has been achieved, blinding, or language of publication.

2.2.5. Exclusion Criteria. The exclusion criteria are as follows: (1) not T2DM patients; (2) the participant is not human; (3) nonoriginal research literature; (4) non-RCT.
2.3. Search Strategy. The English databases (Web of Science, EMBASE, PubMed, and Medline Complete) and Chinese databases (China National Knowledge Infrastructure Databases (CNKI), Chinese Biomedical Database (CBM), Chinese Science and Technology Periodical Database (VIP), and Wan Fang Database) were searched. The search time period is from the establishment of the database to 16th of February, 2020. In addition, the Cochrane Library (until Issue 2, 2020) and clinical trial registration database (ClinicalTrials) were also searched. The search strategy for PubMed is presented in Table S1, as an example.

\subsection{Literature Screening and Study Quality Assessment.} Literature screening and study quality assessment were performed according to the Cochrane system evaluation method. First, the reviewers read the title and abstract for a preliminary screening and then screened them based on the full text. If there is a disagreement, it is resolved through discussion with all researchers. The lack of information would be supplemented by contacting the author through a letter or by imputation [21].

The quality of the literature was evaluated using the Cochrane bias risk assessment tool provided by the Cochrane Collaboration [22], and the following were evaluated: (1) whether the random method is correct; (2) whether the allocation is hidden; (3) blind method; (4) data bias; (5) selective reporting bias; (6) other biases. The evaluation was first conducted independently by two researchers. If there is a disagreement, it is resolved through discussion with all researchers.

2.5. Statistical Analysis. The RevMan version 5.3 statistical software provided by Cochrane Collaboration was used for analysis [23]. When the heterogeneity of RCTs was small $\left(P>0.1, I^{2}<50 \%\right)$, the fixed-effects model was used for meta-analysis. If there is statistical heterogeneity $(P<0.1$, $\left.I^{2}>50 \%\right)$, the reviewers would first look for the source of heterogeneity. If the heterogeneity between the RCTs was statistical rather than clinical heterogeneity, the randomeffects model would be used for meta-analysis. If the heterogeneity was too large or the data source cannot be found, a descriptive analysis would be performed. For continuous variables, the weighted mean difference (WMD) was used as the effect analysis statistic, and the interval is estimated using a $95 \%$ confidence interval $(95 \% \mathrm{CI})$. If the difference in the value of the outcome exceeds 10 times or the unit of measurement is different, the standard MD (SMD) was used. For dichotomous variable, the risk ratio (RR) was used as the effect analysis statistic with 95\% CI.

2.6. Sensitivity Analysis. STATA 15.0 was utilized for sensitivity analysis. The outcomes that met the following conditions were all subjected to sensitivity analysis: (1) random-effects model is used; (2) the results of the fixedeffects model are inconsistent with the results of the random-effects model (whether it is a subgroup result or a summary result). 


\section{Results}

3.1. Results of the Search and Description of Included Trials. A total of 616 articles were retrieved through the database: 590 articles were excluded by reading titles and abstracts, and eight articles were excluded by reading the full text. Finally, 18 articles met the inclusion criteria [15-19, 24-36] (Figure 1). Three studies are by Bo et al. [16, 29, 30], two studies are by Imamura et al. [17], and two studies are by Abdollahi et al. [33, 34], and they were merged. Study characteristics are presented in Table 1.

3.2. Risk of Bias Assessments. The summary and graph of risk of bias are shown in Figure 2.

3.2.1. Random Sequence Generation and Allocation Concealment. Five RCTs $[15,17,26,27,36]$ did not describe the method of generating random sequences and were rated as unclear risk of bias. Other RCTs describe the random sequence generation methods and are assessed as low risk of bias.

Six RCTs [15, 17, 26, 27, 35, 36] did not describe the allocation concealment method and were rated as having an unclear risk of bias. The other RCTs utilized the capsules in the same shape, size, and color to contain curcumin and placebo; hence, they were considered to have allocation concealment and rated as having low risks of bias.

3.2.2. Blinding. Three RCTs $[28,35,36]$ did not specify whether blinding was used and therefore were assessed as high risk of bias. The other RCTs claimed to use blinding, but Goh et al. [25], Brasnyó et al. [26], Bashmakov et al. [27], Zare Javid et al. [15], and Imamura et al. [17] did not describe the implementation process for both researchers and participants. They were rated as unclear risk of bias. The other RCTs described blinding of participants, so the blinding of participants and personnel (performance bias) was rated as low risk of bias.

3.2.3. Incomplete Outcome Data and Selective Reporting. Six RCTs [17, 18, 25, 31, 32, 35] were assessed as unclear risk of bias because of missing data and did not describe whether to use intend-to-treat analysis. The incomplete outcome data of the other RCTs are rated as low risk of bias because the number of missing people and the reasons for the missing between groups is balanced. All RCTs reported study's prespecified outcomes that are of interest in the review; their risks of bias were low.

3.2.4. Other Potential Bias. There were other sources of bias in all RCTs; therefore, the risks of other bias were low.

\subsection{Primary Outcomes}

3.3.1. Homeostasis Model Assessment for Insulin Resistance. Ten RCTs reported the changes in HOMA-IR, and there was a large statistical heterogeneity among the studies
$\left(P<0.00001, I^{2}=83 \%\right)$, so the random-effects model was used. The HOMA-IR of the resveratrol group was significantly lower than that of the control group, and the difference was statistically significant (WMD $=-0.99 ; 95 \% \mathrm{CI}$ $(-1.61,-0.38) ; P=0.002$; random-effect model) (Figure 3).

3.3.2. Total Cholesterol. Ten RCTs reported the changes in TC, and there was a large statistical heterogeneity among the studies $\left(P<0.00001, I^{2}=86 \%\right)$, so the random-effects model was used. The results showed that there was no statistical difference in TC between the resveratrol and control groups (WMD $=-7.11 ; 95 \%$ CI $(-16.28,2.06) ; P=0.13$; randomeffect model) (Figure 4).

3.3.3. Triglyceride. Eleven RCTs reported the changes in TG, and the statistical heterogeneity among the studies was low $\left(P=0.12, I^{2}=34 \%\right)$, so the fixed-effects model was used. The results showed that there was no statistical difference in TG between the resveratrol and control groups $(\mathrm{WMD}=-2.15$; 95\% CI $(-5.52,1.22) ; \quad P=0.21 ;$ fixed-effect model) (Figure 5).

\subsection{Secondary Outcomes}

3.4.1. Glycosylated Hemoglobin. Eleven RCTs reported the changes in $\mathrm{HbA1c}$, and there was a large statistical heterogeneity among the studies $\left(P<0.00001, I^{2}=95 \%\right)$, so the random-effects model was used. The HbAlc of the resveratrol group was significantly lower than that of the control group, and the difference was statistically significant (WMD $=-0.45 ; 95 \%$ CI $(-0.73,-0.16) ; P=0.002$; randomeffect model) (Figure 6).

3.4.2. Fasting Glucose and Fasting Insulin. Fourteen RCTs reported the changes in fasting glucose, and there was a large statistical heterogeneity among the studies $(P<0.00001$, $\left.I^{2}=85 \%\right)$, so the random-effects model was used. The fasting glucose of the resveratrol group was significantly lower than that of the control group, and the difference was statistically significant (WMD $=-19.61 ; 95 \%$ CI $(-26.02,-13.20)$; $P<0.00001$; random-effect model) (Figure 7).

Thirteen RCTs reported the changes in fasting insulin, and there was a large statistical heterogeneity among the studies $\left(P<0.00001, I^{2}=90 \%\right)$, so the random-effects model was used. The fasting insulin of the resveratrol group was significantly lower than that of the control group, and the difference was statistically significant $(\mathrm{SMD}=-0.67 ; 95 \% \mathrm{CI}$ $(1.21,-0.14) ; P=0.01$; random-effect model) (Figure 8).

3.4.3. LDL-C and HDL-C. Ten RCTs reported the changes in LDL-C, and there was a large statistical heterogeneity among the studies $\left(P<0.00001, I^{2}=93 \%\right)$, so the random-effects model was used. The results showed that there was no statistical difference in LDL-C between the resveratrol and control groups $(\mathrm{WMD}=-6.84 ; 95 \%$ CI $(-16.60,2.92)$; $P=0.17$; random-effect model) (Figure 9). 
Records identified through Chinese databases searching $(n=244)$ :

-CNKI (16) -Wan Fang (155) -VIP (27) -CBM (46)
Records identified through searching other language databases $(n=372)$ :

-PubMed (56) -EMBASE (53)

-The Cochrane Library (61)

-Web of Science (45)

-Medline Complete (142)

-ClinicalTrials.gov (15)

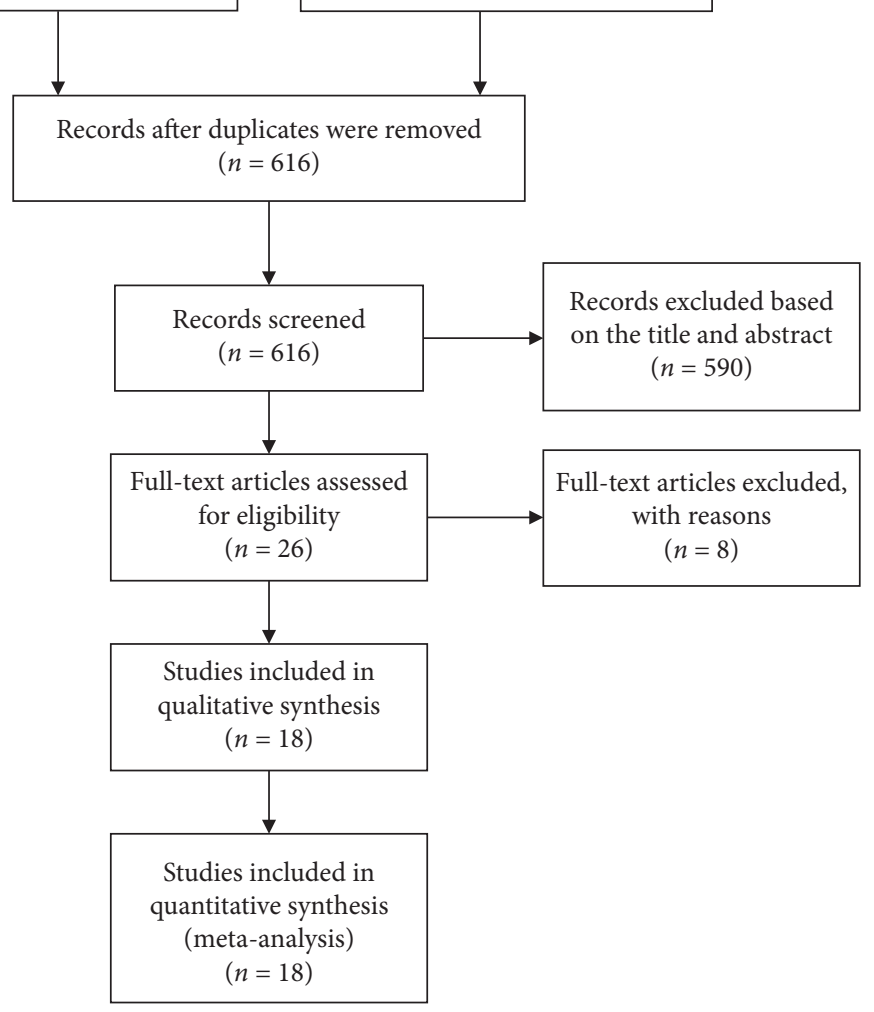

FIgURE 1: Flow diagram of searching and article selection.

TABle 1: The characteristics of the included studies.

\begin{tabular}{|c|c|c|c|c|c|c|c|c|c|}
\hline \multirow{2}{*}{ Study } & \multirow{2}{*}{ Country } & \multicolumn{2}{|c|}{$\begin{array}{c}\text { Sample size } \\
\text { (female/male) }\end{array}$} & \multicolumn{2}{|c|}{ Intervention } & \multirow{2}{*}{$\begin{array}{c}\text { Relevant } \\
\text { outcomes }\end{array}$} & \multicolumn{2}{|c|}{ Mean age (years) } & \multirow{2}{*}{ Duration } \\
\hline & & Trial group & $\begin{array}{l}\text { Control } \\
\text { group }\end{array}$ & Trial group & Control group & & Trial group & $\begin{array}{c}\text { Control } \\
\text { group }\end{array}$ & \\
\hline $\begin{array}{l}\text { Movahed et al. } \\
\text { [23] }\end{array}$ & Iran & $33(16 / 17)$ & $31(17 / 16)$ & $\begin{array}{l}\text { Resveratrol } \\
500 \mathrm{mg} \text {, bid }\end{array}$ & $\begin{array}{l}\text { Microcellulose } \\
\text { (placebo) } \\
500 \mathrm{mg}, \text { bid }\end{array}$ & $\begin{array}{c}\text { HOMA- } \\
\text { IR, } \\
\text { HbA1c, } \\
\text { fasting } \\
\text { blood } \\
\text { glucose, } \\
\text { fasting } \\
\text { insulin, } \\
\text { TC, TG, } \\
\text { HDL-C, } \\
\text { LDL-C }\end{array}$ & $52.45 \pm 6.18$ & $51.81 \pm 6.99$ & $\begin{array}{c}1.5 \\
\text { months }\end{array}$ \\
\hline
\end{tabular}


TABle 1: Continued.

\begin{tabular}{|c|c|c|c|c|c|c|c|c|c|}
\hline \multirow{2}{*}{ Study } & \multirow{2}{*}{ Country } & \multicolumn{2}{|c|}{$\begin{array}{c}\text { Sample size } \\
\text { (female/male) }\end{array}$} & \multicolumn{2}{|c|}{ Intervention } & \multirow{2}{*}{$\begin{array}{l}\text { Relevant } \\
\text { outcomes }\end{array}$} & \multicolumn{2}{|c|}{ Mean age (years) } & \multirow{2}{*}{ Duration } \\
\hline & & Trial group & $\begin{array}{l}\text { Control } \\
\text { group }\end{array}$ & Trial group & Control group & & Trial group & $\begin{array}{l}\text { Control } \\
\text { group }\end{array}$ & \\
\hline Goh et al. [24] & Singapore & $5(0 / 5)$ & $5(0 / 5)$ & $\begin{array}{l}\text { Resveratrol } \\
500 \mathrm{mg}, \mathrm{qd}, \\
\text { initially, } \\
\text { increased by } \\
500 \mathrm{mg} \text { per day } \\
\text { every } 3 \text { days, to } \\
\text { a maximum } \\
\text { dose of } \\
3000 \mathrm{mg} \text { per } \\
\text { day (1000 mg, } \\
\text { tid) }\end{array}$ & $\begin{array}{c}\text { Placebo } \\
500 \mathrm{mg}, \mathrm{qd}, \\
\text { initially, } \\
\text { increased by } \\
500 \mathrm{mg} \text { per day } \\
\text { every } 3 \text { days, to } \\
\text { a maximum } \\
\text { dose of } 3000 \mathrm{mg} \\
\text { per day } \\
(1000 \mathrm{mg}, \mathrm{tid})\end{array}$ & $\begin{array}{c}\text { HbAlc, } \\
\text { fasting } \\
\text { blood } \\
\text { glucose, } \\
\text { fasting } \\
\text { insulin, } \\
\text { TC, TG, } \\
\text { HDL-C, } \\
\text { LDL-C, } \\
\text { adverse } \\
\text { events }\end{array}$ & $55.8 \pm 7.3$ & $56.8 \pm 5.3$ & $\begin{array}{c}3 \\
\text { months }\end{array}$ \\
\hline Brasnyó et al. [25] & Hungary & $10(0 / 10)$ & $9(0 / 9)$ & $\begin{array}{l}\text { Resveratrol } \\
5 \mathrm{mg} \text {, bid }\end{array}$ & Placebo, bid & $\begin{array}{l}\text { HOMA- } \\
\text { IR }\end{array}$ & $57.79 \pm 7.9$ & $52.5 \pm 11.1$ & 1 month \\
\hline $\begin{array}{l}\text { Bashmakov et al. } \\
\text { [26] }\end{array}$ & Egypt & $14(6 / 8)$ & $10(3 / 10)$ & $\begin{array}{l}\text { Resveratrol } \\
50 \mathrm{mg} \text {, bid }\end{array}$ & Placebo, bid & $\begin{array}{l}\text { Fasting } \\
\text { blood } \\
\text { glucose, } \\
\text { fasting } \\
\text { insulin, } \\
\text { TC, } \\
\text { HDL-C, } \\
\text { LDL-C }\end{array}$ & $54.0 \pm 10.1$ & $59.8 \pm 6.6$ & $\begin{array}{c}2 \\
\text { months }\end{array}$ \\
\hline Bhatt et al. [27] & India & $28(20 / 9)$ & $29(16 / 12)$ & $\begin{array}{c}\text { Resveratrol } \\
250 \mathrm{mg}, \mathrm{qd}, \\
\text { with oral } \\
\text { hypoglycemic } \\
\text { agents such as } \\
\text { glibenclamide } \\
\text { and/or } \\
\text { metformin }\end{array}$ & $\begin{array}{l}\text { Oral } \\
\text { hypoglycemic } \\
\text { agents such as } \\
\text { glibenclamide } \\
\text { and/or } \\
\text { metformin }\end{array}$ & $\begin{array}{l}\text { HbAlc, } \\
\text { fasting } \\
\text { blood } \\
\text { glucose, } \\
\text { TC, TG, } \\
\text { HDL-C, } \\
\text { LDL-C }\end{array}$ & $56.67 \pm 8.91$ & $57.75 \pm 8.71$ & $\begin{array}{c}3 \\
\text { months }\end{array}$ \\
\hline $\begin{array}{l}\text { Zare Javid et al. } \\
\text { [15] }\end{array}$ & Iran & $21(18 / 4)$ & $22(16 / 5)$ & $\begin{array}{l}\text { Resveratrol } \\
240 \mathrm{mg} \text {, bid }\end{array}$ & $\begin{array}{c}\text { Starch } \\
\text { (placebo) } \\
240 \mathrm{mg} \text {, bid }\end{array}$ & $\begin{array}{l}\text { HOMA- } \\
\text { IR, } \\
\text { fasting } \\
\text { blood } \\
\text { glucose, } \\
\text { fasting } \\
\text { insulin, } \\
\text { TG }\end{array}$ & $49.1 \pm 7.4$ & $50.9 \pm 8.9$ & 1 month \\
\hline $\begin{array}{l}\text { Bo et al. }[16,28 \text {, } \\
29]\end{array}$ & Italy & $130(51 / 79)$ & $62(15 / 47)$ & $\begin{array}{l}\text { Resveratrol } \\
500 \mathrm{mg} \text {, qd, or } \\
40 \mathrm{mg} \text {, qd }\end{array}$ & Placebo, qd & $\begin{array}{l}\text { HOMA- } \\
\text { IR, } \\
\text { HbAlc, } \\
\text { fasting } \\
\text { blood } \\
\text { glucose, } \\
\text { fasting } \\
\text { insulin, } \\
\text { TC, TG, } \\
\text { HDL-C, } \\
\text { LDL-C }\end{array}$ & $64.95 \pm 8.08$ & $65.4 \pm 8.8$ & $\begin{array}{c}6 \\
\text { months }\end{array}$ \\
\hline $\begin{array}{l}\text { Imamura et al. } \\
{[17]}\end{array}$ & Japan & $25(10 / 15)$ & $25(14 / 11)$ & $\begin{array}{l}\text { Resveratrol } \\
100 \mathrm{mg} \text {, qd }\end{array}$ & Placebo, qd & $\begin{array}{l}\text { HbAlc, } \\
\text { fasting } \\
\text { blood } \\
\text { glucose, } \\
\text { fasting } \\
\text { insulin, } \\
\text { TC, TG, } \\
\text { HDL-C, } \\
\text { adverse } \\
\text { events }\end{array}$ & $57.4 \pm 10.6$ & $58.2 \pm 10.1$ & $\begin{array}{c}3 \\
\text { months }\end{array}$ \\
\hline
\end{tabular}


Table 1: Continued.

\begin{tabular}{|c|c|c|c|c|c|c|c|c|c|}
\hline \multirow{2}{*}{ Study } & \multirow{2}{*}{ Country } & \multicolumn{2}{|c|}{$\begin{array}{c}\text { Sample size } \\
\text { (female/male) }\end{array}$} & \multicolumn{2}{|c|}{ Intervention } & \multirow{2}{*}{$\begin{array}{l}\text { Relevant } \\
\text { outcomes }\end{array}$} & \multicolumn{2}{|c|}{ Mean age (years) } & \multirow{2}{*}{ Duration } \\
\hline & & Trial group & $\begin{array}{l}\text { Control } \\
\text { group }\end{array}$ & Trial group & Control group & & Trial group & $\begin{array}{l}\text { Control } \\
\text { group }\end{array}$ & \\
\hline $\begin{array}{l}\text { Sattarinezhad et } \\
\text { al. [18] }\end{array}$ & Iran & $30(16 / 14)$ & $30(17 / 13)$ & $\begin{array}{l}\text { Resveratrol } \\
500 \mathrm{mg}, \\
\mathrm{qd}+\text { losartan } \\
12.5 \mathrm{mg}, \mathrm{qd}\end{array}$ & $\begin{array}{c}\text { Placebo } \\
500 \mathrm{mg}, \\
\text { qd + losartan } \\
12.5 \mathrm{mg}, \mathrm{qd}\end{array}$ & $\begin{array}{l}\text { HOMA- } \\
\text { IR, } \\
\text { HbA1c, } \\
\text { fasting } \\
\text { blood } \\
\text { glucose, } \\
\text { fasting } \\
\text { insulin, } \\
\text { adverse } \\
\text { events }\end{array}$ & $56.8 \pm 9.7$ & $55.7 \pm 10.8$ & $\begin{array}{c}3 \\
\text { months }\end{array}$ \\
\hline $\begin{array}{l}\text { Khodabandehloo } \\
\text { et al. [19] }\end{array}$ & Iran & $25(12 / 13)$ & $20(10 / 10)$ & $\begin{array}{l}\text { Resveratrol } \\
400 \mathrm{mg} \text {, bid }\end{array}$ & $\begin{array}{c}\text { Microcellulose } \\
\text { (placebo) } \\
400 \mathrm{mg}, \text { bid }\end{array}$ & $\begin{array}{c}\text { HOMA- } \\
\text { IR, } \\
\text { HbAlc, } \\
\text { fasting } \\
\text { blood } \\
\text { glucose, } \\
\text { fasting } \\
\text { insulin, } \\
\text { TC, TG, } \\
\text { HDL-C, } \\
\text { LDL-C, } \\
\text { adverse } \\
\text { events }\end{array}$ & $56.48 \pm 6.72$ & $61.10 \pm 5.61$ & $\begin{array}{c}2 \\
\text { months }\end{array}$ \\
\hline $\begin{array}{l}\text { Seyyedebrahimi } \\
\text { et al. [30] }\end{array}$ & Iran & $23(12 / 11)$ & $23(13 / 10)$ & $\begin{array}{l}\text { Resveratrol } \\
400 \mathrm{mg} \text {, bid }\end{array}$ & $\begin{array}{c}\text { Microcellulose } \\
\text { (placebo) } \\
400 \mathrm{mg}, \text { bid }\end{array}$ & $\begin{array}{l}\text { HOMA- } \\
\text { IR, } \\
\text { HbA1c, } \\
\text { fasting } \\
\text { blood } \\
\text { glucose, } \\
\text { fasting } \\
\text { insulin, } \\
\text { TC, TG, } \\
\text { HDL-C, } \\
\text { LDL-C, } \\
\text { MDA, } \\
\text { adverse } \\
\text { events }\end{array}$ & $54.96 \pm 6.37$ & $58.72 \pm 6.06$ & $\begin{array}{c}2 \\
\text { months }\end{array}$ \\
\hline Hoseini et al. [31] & Iran & $\begin{array}{c}28 \\
\text { (unknown/ } \\
\text { unknown) }\end{array}$ & $\begin{array}{c}28 \\
\text { (unknown/ } \\
\text { unknown) }\end{array}$ & $\begin{array}{l}\text { Resveratrol } \\
500 \mathrm{mg} \text {, qd }\end{array}$ & Placebo, qd & $\begin{array}{c}\text { HOMA- } \\
\text { IR, } \\
\text { fasting } \\
\text { blood } \\
\text { glucose, } \\
\text { fasting } \\
\text { insulin, } \\
\text { TG, TC, } \\
\text { HDL-C, } \\
\text { LDL-C, } \\
\text { MDA, } \\
\text { TAC, } \\
\text { adverse } \\
\text { events }\end{array}$ & $61.0 \pm 8.6$ & $63.3 \pm 10.1$ & 1 month \\
\hline
\end{tabular}


TABLE 1: Continued.

\begin{tabular}{|c|c|c|c|c|c|c|c|c|c|}
\hline \multirow{2}{*}{ Study } & \multirow{2}{*}{ Country } & \multicolumn{2}{|c|}{$\begin{array}{c}\text { Sample size } \\
\text { (female/male) }\end{array}$} & \multicolumn{2}{|c|}{ Intervention } & \multirow{2}{*}{$\begin{array}{l}\text { Relevant } \\
\text { outcomes }\end{array}$} & \multicolumn{2}{|c|}{ Mean age (years) } & \multirow{2}{*}{ Duration } \\
\hline & & Trial group & $\begin{array}{l}\text { Control } \\
\text { group }\end{array}$ & Trial group & Control group & & Trial group & $\begin{array}{l}\text { Control } \\
\text { group }\end{array}$ & \\
\hline $\begin{array}{l}\text { Abdollahi et al. } \\
{[32,33]}\end{array}$ & Iran & $35(15 / 20)$ & $36(16 / 20))$ & $\begin{array}{l}\text { Resveratrol } \\
500 \mathrm{mg} \text {, bid }\end{array}$ & Placebo, bid & $\begin{array}{l}\text { HOMA- } \\
\text { IR, } \\
\text { HbA1c, } \\
\text { fasting } \\
\text { blood } \\
\text { glucose, } \\
\text { fasting } \\
\text { insulin, } \\
\text { TG, TC, } \\
\text { HDL-C, } \\
\text { LDL-C, } \\
\text { adverse } \\
\text { events }\end{array}$ & $50.14 \pm 7.38$ & $50.06 \pm 7.69$ & $\begin{array}{c}2 \\
\text { months }\end{array}$ \\
\hline Zhang et al. [34] & China & $48(24 / 24)$ & $48(23 / 25)$ & $\begin{array}{l}\text { Resveratrol } \\
300 \mathrm{mg} \text {, bid }\end{array}$ & Blank & $\begin{array}{c}\text { HbAlc, } \\
\text { fasting } \\
\text { blood } \\
\text { glucose, } \\
\text { fasting } \\
\text { insulin, } \\
\text { TG, TC, } \\
\text { HDL-C, } \\
\text { LDL-C }\end{array}$ & $50.9 \pm 9.7$ & $52.3 \pm 11.2$ & $\begin{array}{c}3 \\
\text { months }\end{array}$ \\
\hline Ying et al. [35] & China & $32(12 / 20)$ & $31(11 / 20)$ & $\begin{array}{l}\text { Resveratrol } \\
500 \mathrm{mg} \text {, bid }\end{array}$ & Blank & $\begin{array}{l}\text { HbA1c, } \\
\text { fasting } \\
\text { blood } \\
\text { glucose, } \\
\text { fasting } \\
\text { insulin }\end{array}$ & $64.94 \pm 1.36$ & $64.95 \pm 1.35$ & $\begin{array}{c}2 \\
\text { months }\end{array}$ \\
\hline
\end{tabular}

HOMA-IR: homeostasis model assessment for insulin resistance; TC: total cholesterol; TG: triglyceride; LDL-C: low density lipoprotein cholesterol; HDL-C: high density lipoprotein cholesterol.

Eleven RCTs reported the changes in HDL-C, and there was a large statistical heterogeneity among the studies $\left(P<0.0001, I^{2}=72 \%\right)$, so the random-effects model was used. The results showed that there was no statistical difference in HDL-C between the resveratrol and control groups (WMD $=1.38$; $95 \% \mathrm{CI}(-0.43,3.18) ; P=0.13$; random-effect model) (Figure 10).

3.4.4. Oxidative-Stress-Related Indicators. Two RCTs reported the changes in MDA, and the statistical heterogeneity among the studies was low $\left(P=0.55, I^{2}=0 \%\right)$, so the fixed-effects model was used. The results showed that there was no statistical difference in MDA between the resveratrol and control groups $(\mathrm{WMD}=-0.05 ; 95 \%$ CI $(-0.33,0.23)$; $P=0.71$; fixed-effect model) (Figure 11).

3.5. Adverse Events. Two RCTs reported the adverse events, and the statistical heterogeneity among the studies was low $\left(P=0.51, I^{2}=0 \%\right)$, so the fixed-effects model was used. The results showed that there was no statistical difference of adverse events between the resveratrol and control groups $(\mathrm{RR}=2 ; 95 \% \mathrm{CI}(0.44,9.03) ; P=0.37$; fixed-effect model $)]$ (Figure 12).
3.6. Sensitivity Analysis Results. Sensitivity analyses were performed for five outcomes: TC and LDL-C. (1) In the outcome "TC," no matter which study was removed, the results were not significantly changed, suggesting that the heterogeneity may not come from RCT (Figure 13(a)). (2) In the outcome "LDL-C," after we omitted the study of Zhang et al. [35], we found that the estimate of the result moved out of the lower limit of 95\% CI (Figure 13(b)). This indicates that the study of Zhang et al. [35] may be the source of heterogeneity of LDL-C outcomes.

\section{Discussion}

This systematic review and meta-analysis included 15 RCTs involving 896 patients. This research showed that resveratrol may improve HOMA-IR and reduce HbAlc, fasting blood sugar, and fasting insulin levels, indicating that resveratrol may reduce insulin resistance, thereby lowering blood sugar and insulin levels. Although the results found in the current research are meaningful, they should be interpreted with caution due to the high heterogeneity of these results and small number of participants involved. This study did not show the positive effects of resveratrol on blood lipid levels and oxidative stress levels but showed that they have a trend of improvement. In the future, more RCTs may be needed to 


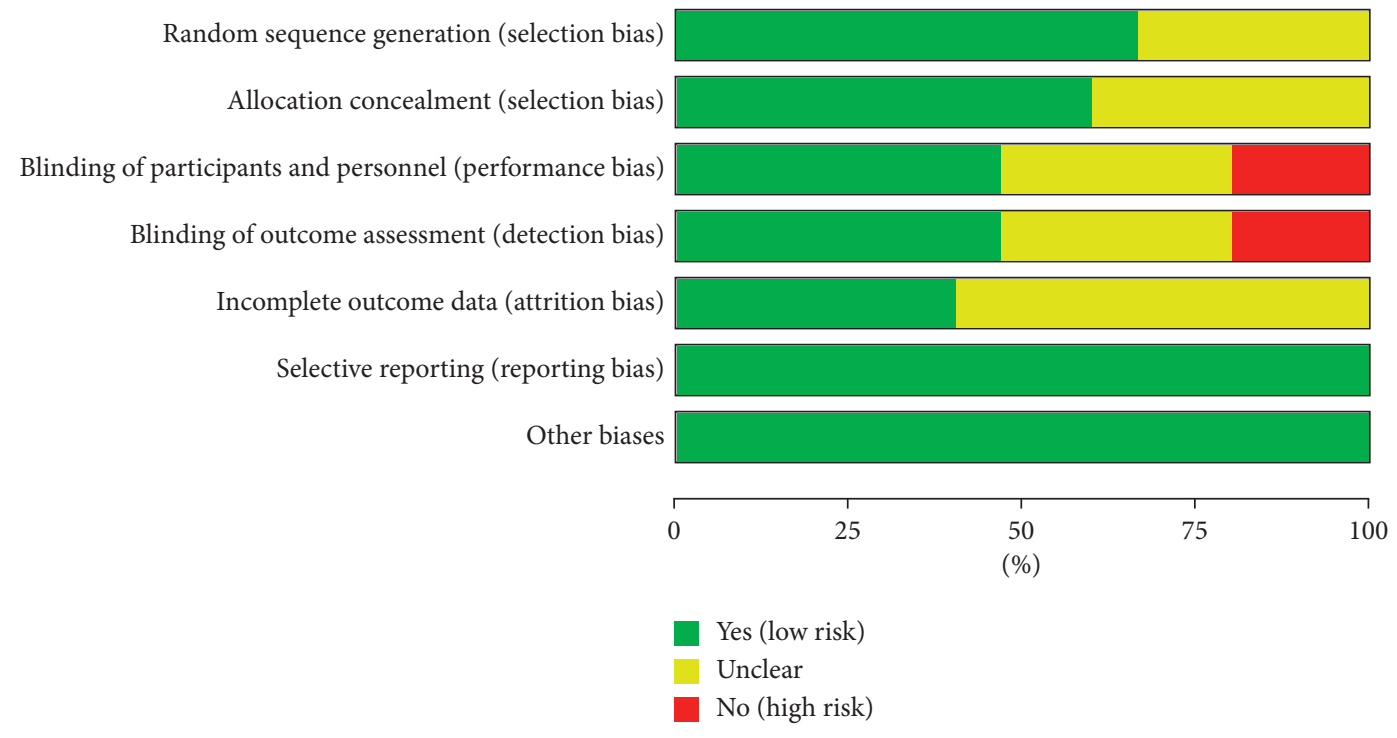

(a)

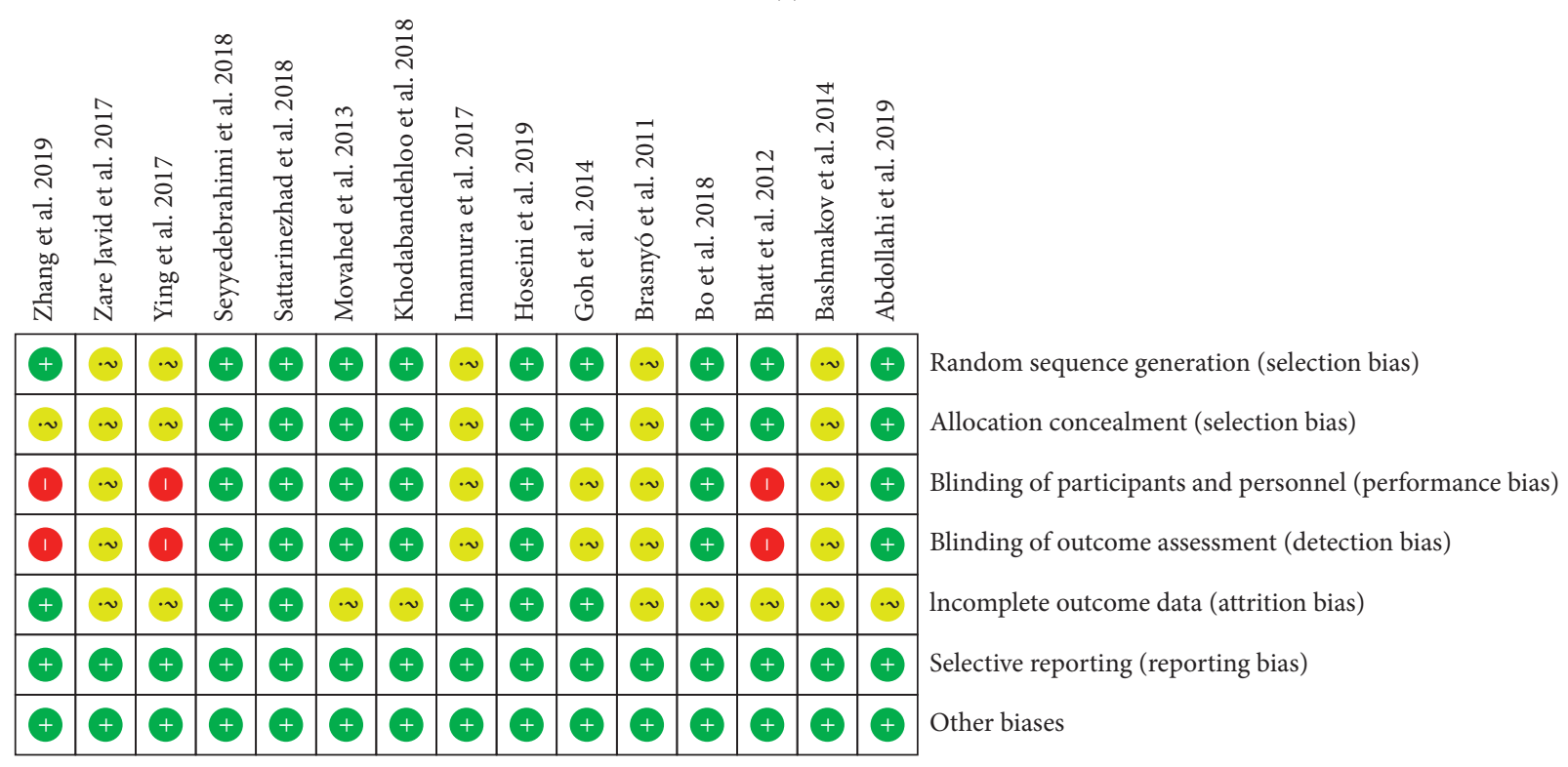

(b)

Figure 2: The risk of bias assessment. (a) The risk of bias graph; (b) the risk of bias table.

confirm or modify the effects of resveratrol on blood lipids and oxidative stress indicators in patients with T2DM. Only two RCTs reported adverse events, and the meta-analysis results showed that there was no statistically significant difference in adverse events between the control and resveratrol groups. Due to the insufficient number of RCTs, this result is doubtful. It can only be inferred based on the existing evidence that resveratrol may be a safe therapy for the treatment of T2DM. More RCTs are needed in the future to report on the safety of resveratrol.

Resveratrol, as a type of polyphenolic phytoalexin, has good antioxidant properties. It is produced by plants under the action of exogenous stimuli, such as ultraviolet light irradiation, mechanical damage or fungal infection [37-41]. A large number of in vitro and in vivo tests have shown that resveratrol can effectively prevent hypertension through antioxidant effects [42], cardiovascular diseases [43], nonalcoholic fatty liver [44], metabolic syndrome [45], aging [46], cancer [47], and immunological diseases [48], through its antioxidant effect, and has a good application prospect. Based on this, the research on the safety of resveratrol is meaningful. Williams et al. [49] showed that resveratrol is not irritating to the skin and eyes, and the micronucleus test in vivo proved that resveratrol has no genetic toxicity. After a 90-day subchronic toxicity test, it was found that resveratrol did not cause any adverse effects on the body and did not have reproductive toxicity at the maximum dose of $700 \mathrm{mg} /$ (kg.d). This preliminarily proves that resveratrol is nontoxic and safe. Hebbar et al. [50] administered resveratrol to CD rats at $0.3,1.0$, and $3.0 \mathrm{~g} /(\mathrm{kg} \cdot \mathrm{d})$. They found that, at $0.3 \mathrm{~g} /$ 


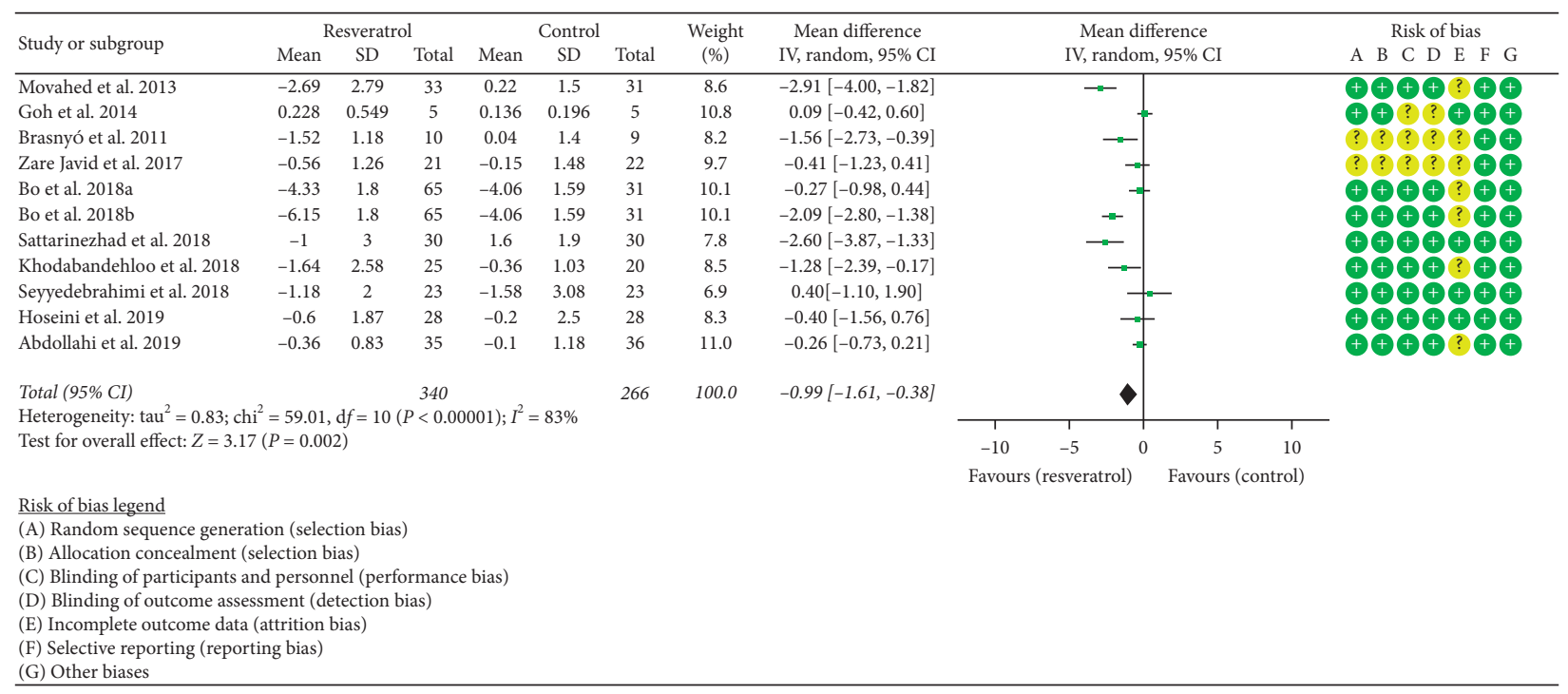

FIGURE 3: Homeostasis model assessment for insulin resistance (HOMA-IR).

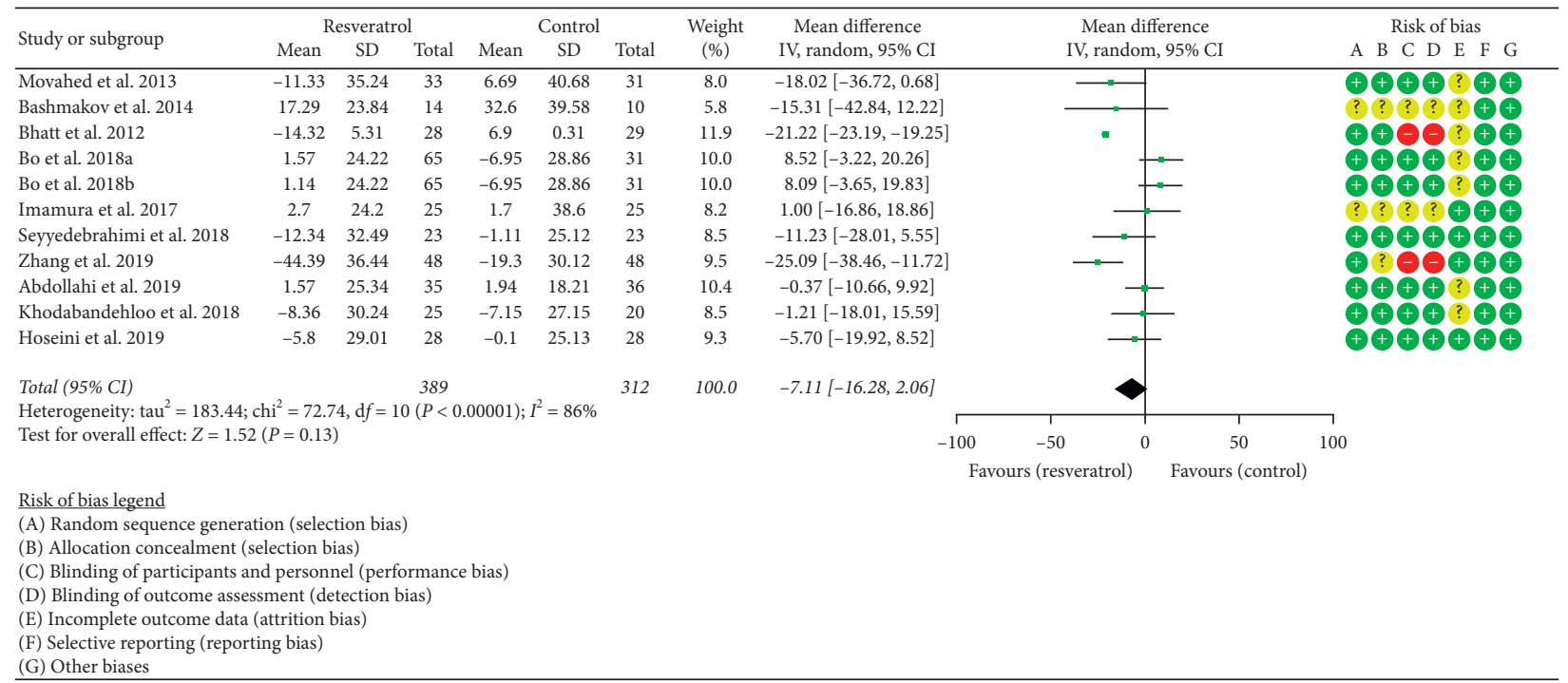

FIgURE 4: Total cholesterol.

\begin{tabular}{|c|c|c|c|c|c|c|c|c|c|c|}
\hline \multirow{2}{*}{ Study or subgroup } & \multicolumn{3}{|c|}{ Resveratrol } & \multicolumn{3}{|c|}{ Control } & \multirow{2}{*}{$\begin{array}{c}\text { Weight } \\
(\%)\end{array}$} & \multirow{2}{*}{$\begin{array}{l}\text { Mean difference } \\
\text { IV, fixed, } 95 \% \text { CI }\end{array}$} & \multirow{2}{*}{$\begin{array}{l}\text { Mean difference } \\
\text { IV, fixed, } 95 \% \text { CI }\end{array}$} & Risk of bias \\
\hline & Mean & SD & Total & Mean & SD & Total & & & & 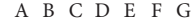 \\
\hline Movahed et al. 2013 & -17.81 & 39.87 & 33 & -11.56 & 36.71 & 31 & 3.2 & $-6.25[-25.01,12.51]$ & & $\odot+\odot+?+\odot$ \\
\hline Goh et al. 2014 & 32.76 & 66.41 & 5 & -1.77 & 43.39 & 5 & 0.2 & $34.53[-35.00,104.06]$ & & $+\oplus$ \\
\hline Bhatt et al. 2012 & 0.75 & 9.59 & 28 & 2.8 & 2.96 & 29 & 82.6 & $-2.05[-5.76,1.66]$ & & $\oplus \oplus$ \\
\hline Zare Javid et al. 2017 & -12 & 65.56 & 21 & 9.3 & 67.71 & 22 & 0.7 & $-21.30[-61.13,18.53]$ & & $\oplus \oplus+$ \\
\hline Bo et al. 2018a & 23.78 & 48.53 & 65 & 3.68 & 44.13 & 31 & 3.0 & $20.10[0.59,39.61]$ & - & $\oplus+$ \\
\hline Bo et al. 2018b & -8.76 & 48.53 & 65 & 3.68 & 44.13 & 31 & 3.0 & $-12.44[-31.95,7.07]$ & - & ? ? ? ? $\odot \oplus$ \\
\hline Imamura et al. 2017 & 3.4 & 47.2 & 25 & -2.4 & 75.4 & 25 & 0.9 & $5.80[-29.07,40.67]$ & + & $\oplus+$ \\
\hline Zhang et al. 2019 & -69.44 & 121.25 & 48 & -3.472 & 129.77 & 48 & 0.5 & $-65.97[-116.21,-15.73]$ & — & \\
\hline Khodabandehloo et al. 2018 & -1.92 & 67.19 & 25 & -19.44 & 49.02 & 20 & 1.0 & $17.52[-16.47,51.51]$ & $f-$ & \\
\hline Abdollahi et al. 2019 & -0.54 & 55.99 & 35 & 2.05 & 34.98 & 36 & 2.4 & $-2.59[-24.38,19.20]$ & . & \\
\hline Hoseini et al. 2019 & -7.4 & 60.51 & 28 & 2.4 & 57.2 & 28 & 1.2 & $-9.80[-40.64,21.04]$ & $-t$ & \\
\hline Seyyedebrahimi et al. 2018 & -9.95 & 62.57 & 23 & 2.88 & 38.63 & 23 & 1.3 & $-12.83[-42.88,17.22]$ & - & \\
\hline \multirow{2}{*}{\multicolumn{4}{|c|}{$\begin{array}{l}\text { Total }(95 \% \mathrm{CI}) \\
\text { Heterogeneity: } \mathrm{chi}^{2}=16.62, \mathrm{~d} f=11(P=0.12) ; I^{2}=34 \% \\
\text { Test for overall effect: } Z=1.25(P=0.21)\end{array}$}} & & & 329 & 100.0 & $-2.15[-5.52,1.22]$ & & \\
\hline & & & & & & & & & $\begin{array}{cccccc} & 1 & & 1 & 1 \\
-200 & -100 & 0 & 100 & 200\end{array}$ & \\
\hline $\begin{array}{l}\text { Risk of bias legend } \\
\text { (A) Random sequence gener } \\
\text { (B) Allocation concealment } \\
\text { (C) Blinding of participants } \\
\text { (D) Blinding of outcome asse } \\
\text { (E) Incomplete outcome data } \\
\text { (F) Selective reporting (repor } \\
\text { (G) Other biases }\end{array}$ & $\begin{array}{l}\text { ion (selec } \\
\text { lection b } \\
\text { d person } \\
\text { ment (de } \\
\text { attrition } \\
\text { ng bias) }\end{array}$ & $\begin{array}{l}\text { tion bias } \\
\text { ias) } \\
\text { nel (perf } \\
\text { tection } \\
\text { bias) }\end{array}$ & $\begin{array}{l}\text { ormance } \\
\text { ias) }\end{array}$ & bias) & & & & & Favours (resveratrol) & \\
\hline
\end{tabular}




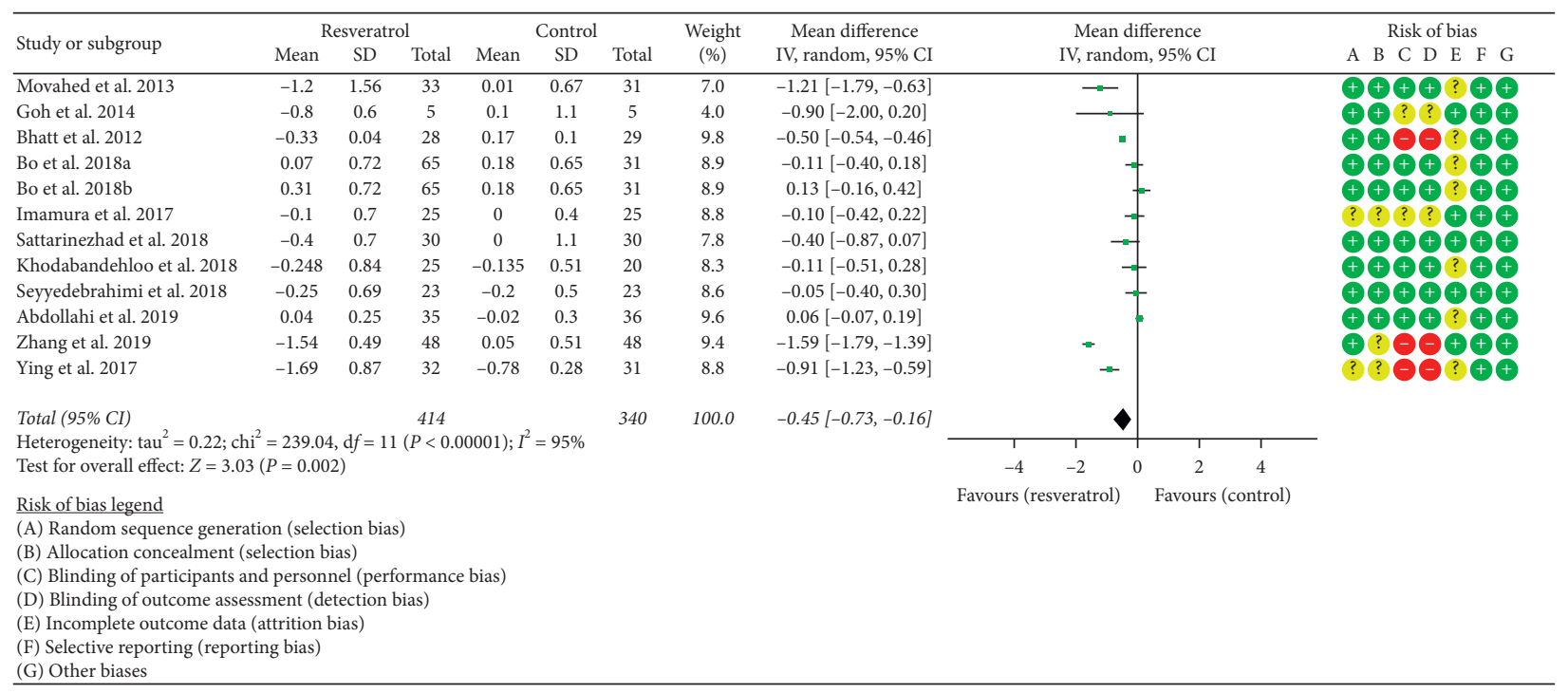

Figure 6: Glycosylated hemoglobin.

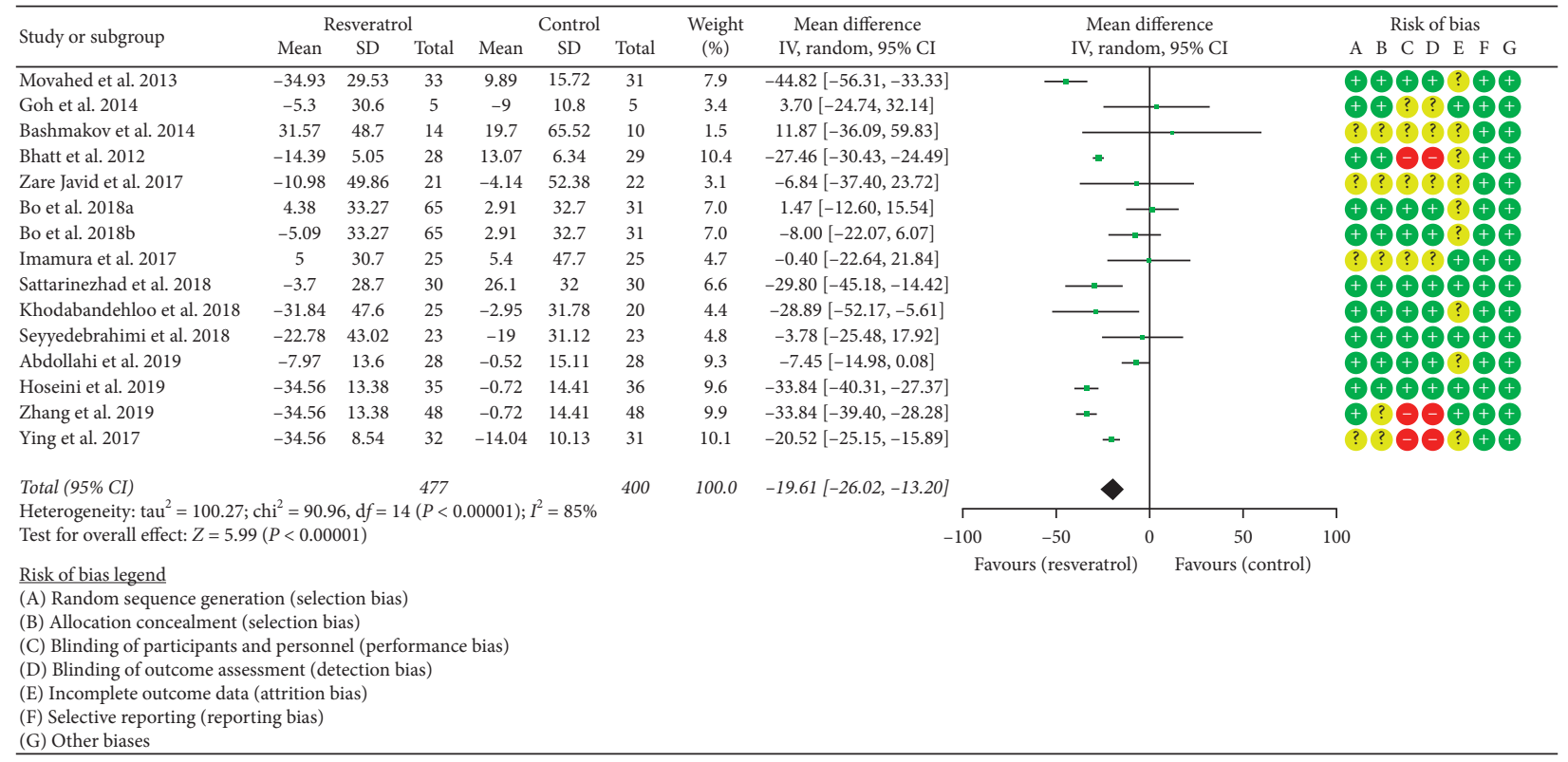

Figure 7: Fasting blood glucose.

$(\mathrm{kg} \cdot \mathrm{d})$, the rats showed no adverse reactions. However, at 1.0 and $3.0 \mathrm{~g} /(\mathrm{kg} \cdot \mathrm{d})$, female and male rats experienced different degrees of dehydration, dyspnea, kidney toxicity, and increased serum liver enzymes. It shows that resveratrol has certain toxicity at high doses. In order to determine the safe dose range of resveratrol, Johnson et al. [51] also studied the subchronic oral toxicity of resveratrol. The results showed that when the dose was increased to $1000 \mathrm{mg} /(\mathrm{kg} \cdot \mathrm{d})$, resveratrol showed certain toxicity; it showed that the noobserved-adverse-effect levels (NOAELs) of resveratrol in rats and dogs are $200 \mathrm{mg} /(\mathrm{kg} \cdot \mathrm{d})$ and $600 \mathrm{mg} /(\mathrm{kg} \cdot \mathrm{d})$, respectively. Since the content of resveratrol in plants or foods is lower than NOAEL, it can be considered that normal consumption of foods rich in resveratrol can not only give full play to its physiological activities but also be safe. In clinical trials, a randomized, double-blind, placebo-controlled clinical trial found that, at a clinical dose of $150 \mathrm{mg} /$ day, no effect of resveratrol supplementation on cardiometabolic risk parameters was observed. It suggests that resveratrol supplements are well tolerated and safe [52]. Federica et al. found that high daily doses ( $\geq 300 \mathrm{mg} /$ day) of resveratrol can promote cardiovascular health. Resveratrol is well tolerated, and no serious adverse events occurred in most eligible trials [53]. This study also showed that the adverse events of the resveratrol group were the same as those of the control group, and no serious adverse events occurred, which suggested that resveratrol has good safety.

Most of the results, such as HOMA-IR, TC, TG, and LDL-C, have large heterogeneity, so this study used sensitivity analysis to find the source of heterogeneity. Sensitivity 


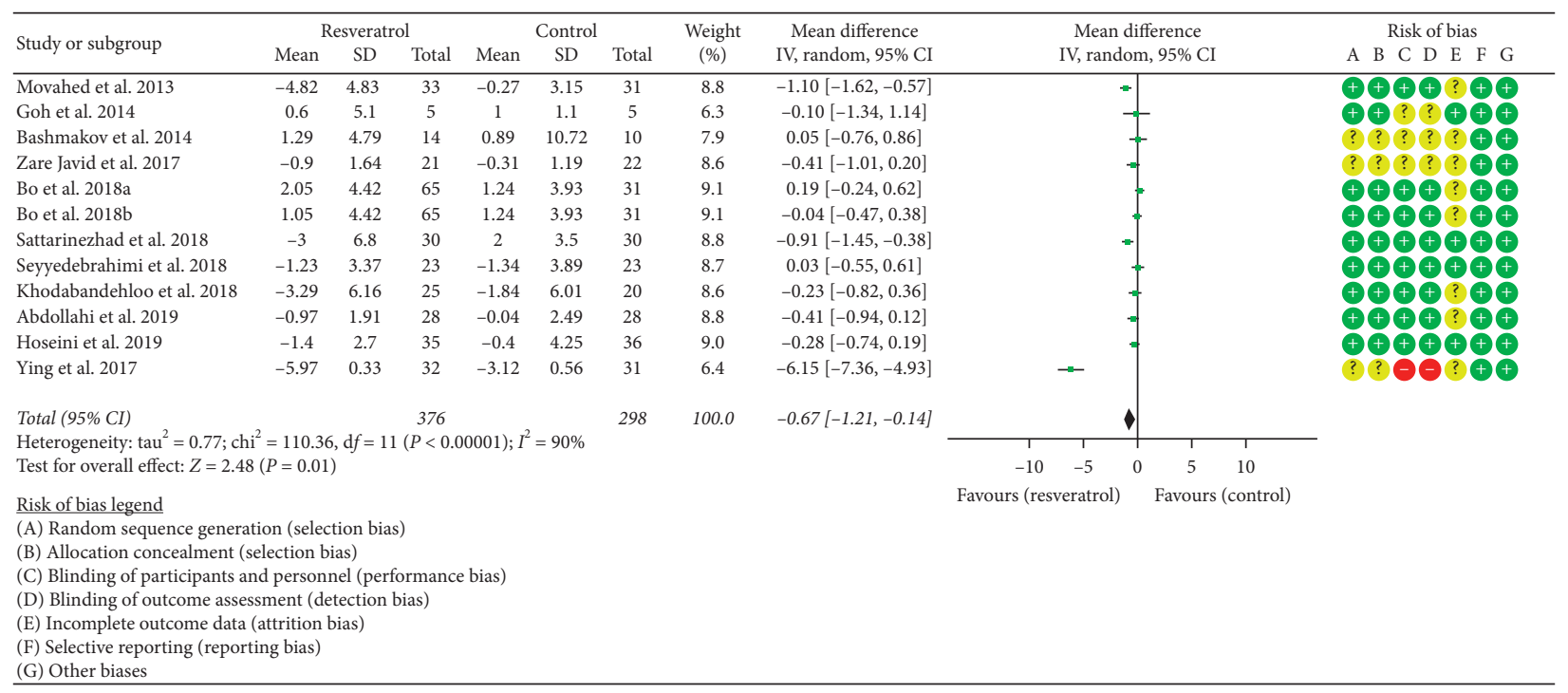

FIgURE 8: Fasting insulin.

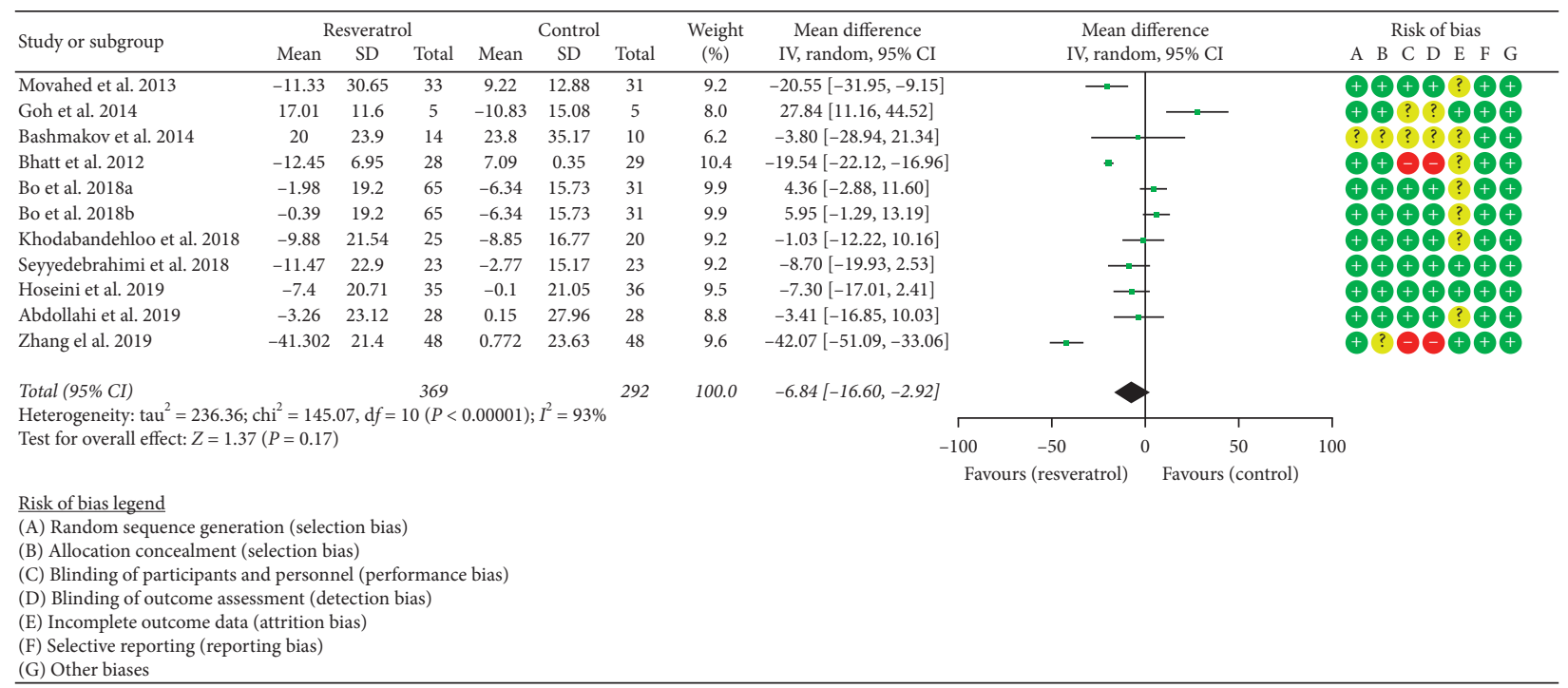

Figure 9: LDL-C.

analyses were performed for TC and LDL-C. For outcome "LDL-C," the study of Zhang et al. [35] may be the source of heterogeneity. Compared with other studies, the blinding method of Zhang et al. [35] was assessed as a high risk of bias, and allocation concealment was assessed as an unknown risk of bias. This suggests that the heterogeneity may be due to the low quality of RCTs, and failure to apply blinding may lead to biased results. In addition to the possible sources of heterogeneity found by sensitivity analysis, heterogeneity may also come from ethnic differences, regional differences, gender differences, and so on: (1) most of the RCTs are from Iran, and a few are from China, Japan, Egypt, Singapore, Hungary, and Italy (see Table 1); there are differences between races in these countries, and this may cause different sensitivities to resveratrol. (2) The gender composition ratio of RCTs is different. The participants in the studies of Goh et al. [25] and Brasnyó et al. [26] were all male, while the gender ratio in the study of Hoseini et al. [32] is not clear. Males and females having different sensitivities to drugs may lead to heterogeneity. (3) The dosage, preparation type, and usage of resveratrol in RCTs are different. The difference between the dosage and type of preparation may affect the efficacy of the drug, which may be the source of heterogeneity.

This meta-analysis is similar to the works of Zhu et al. [13] and Liu et al. [14] in that both have shown that resveratrol can improve HOMA-IR, fasting blood glucose, and $\mathrm{HbA} 1 \mathrm{c}$ insulin levels. The differences are as follows. (1) Research Process. This research was registered with PROSPERO in advance, and it was analyzed strictly according to the protocol and PRISMA-guidelines. The inclusion and exclusion criteria were more stringent. (2) Literature Quality 


\begin{tabular}{|c|c|c|c|c|c|c|c|c|c|c|c|c|c|}
\hline \multirow{3}{*}{$\begin{array}{l}\text { Study or subgroup } \\
\text { Movahed et al. } 2013\end{array}$} & \multicolumn{3}{|c|}{ Resveratrol } & \multicolumn{3}{|c|}{ Control } & \multirow{3}{*}{$\begin{array}{c}\begin{array}{c}\text { Weight } \\
(\%)\end{array} \\
10.4\end{array}$} & \multirow{3}{*}{$\begin{array}{c}\text { Mean difference } \\
\text { IV, random, 95\% CI }\end{array}$} & \multirow{2}{*}{\multicolumn{3}{|c|}{$\begin{array}{l}\text { Mean difference } \\
\text { IV, random, } 95 \% \text { CI }\end{array}$}} & \multicolumn{2}{|r|}{ Risk of bias } \\
\hline & \multirow{2}{*}{$\begin{array}{c}\text { Mean } \\
4.75\end{array}$} & \multirow{2}{*}{$\begin{array}{c}\mathrm{SD} \\
5.83\end{array}$} & \multirow{2}{*}{$\begin{array}{c}\text { Total } \\
33\end{array}$} & \multirow{2}{*}{$\begin{array}{c}\text { Mean } \\
-2.4\end{array}$} & \multirow{2}{*}{$\begin{array}{c}\mathrm{SD} \\
6.26\end{array}$} & \multirow{2}{*}{$\begin{array}{c}\text { Total } \\
31\end{array}$} & & & & & & & A $B$ B $C D E A B$ \\
\hline & & & & & & & & & & & - & & $+\odot \odot+?+\odot$ \\
\hline Goh et al. 2014 & -2.32 & 7.73 & 5 & 1.93 & 4.64 & 5 & 3.8 & $-4.25[-12.15,3.65]$ & & 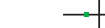 & + & & $+\oplus$ \\
\hline Bashmakov et al. 2014 & -1.43 & 8.39 & 14 & 3.4 & 4.91 & 10 & 6.4 & $-4.83[-10.18,0.52]$ & & - & & & ? ? ? ? ? $\oplus \oplus$ \\
\hline Bhatt et al. 2012 & -2.03 & 0.9 & 28 & -2 & 2.59 & 29 & 13.9 & $-0.03[-1.03,0.97]$ & & & & & $\oplus \odot$ \\
\hline Bo et al. 2018a & 0.83 & 17.01 & 65 & 0.36 & 4.91 & 31 & 7.7 & $0.47[-4.01,4.95]$ & & & 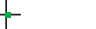 & & $\oplus$ \\
\hline Bo et al. 2018b & 1.48 & 17.01 & 65 & 0.36 & 4.91 & 31 & 7.7 & $1.12[-3.36,5.60]$ & & & - & & $\oplus+$ \\
\hline Imamura et al. 2017 & -2.4 & 75.4 & 25 & 0.6 & 6.8 & 25 & 0.4 & $-3.00[-32.68,26.68]$ & & & & & ? ? ? ? $\oplus \oplus \oplus$ \\
\hline Khodabandehloo et al. 2018 & -1.96 & 2.28 & 25 & -1.1 & 4.86 & 20 & 11.8 & $-0.86[-3.17,1.45]$ & & & & & $\oplus+$ \\
\hline Seyyedebrahimi et al. 2018 & -2.39 & 3.78 & 23 & -2.05 & 4.27 & 23 & 11.7 & $-0.34[-2.67,1.99]$ & & & & & \\
\hline Hoseini et al. 2019 & 3.1 & 7.18 & 35 & -0.4 & 5.67 & 36 & 10.3 & $3.50[0.49,6.51]$ & & & - & & \\
\hline Abdollahi et al. 2019 & 3.62 & 8.75 & 28 & -0.16 & 5.75 & 28 & 8.7 & $3.78[-0.10,7.66]$ & & & $=-$ & & \\
\hline Zhang et al. 2019 & 6.948 & 12.27 & 48 & 0.772 & 11.66 & 48 & 7.2 & $6.18[1.39,10.96]$ & & & - & & \\
\hline $\begin{array}{l}\text { Total }(95 \% \text { CI }) \\
\end{array}$ & $=3918$ & $d f=11$ & $\begin{array}{c}394 \\
P=00\end{array}$ & & & 317 & 100.0 & $1.38[-0.43,3.18]$ & & & & & \\
\hline Test for overall effect: $Z=1.4$ & $P=0.13$ & & & & & & & & -50 & -25 & 25 & 50 & \\
\hline $\begin{array}{l}\text { Risk of bias legend } \\
\text { (A) Random sequence gener } \\
\text { (B) Allocation concealment } \\
\text { (C) Blinding of participants } \\
\text { (D) Blinding of outcome asse } \\
\text { (E) Incomplete outcome data } \\
\text { (F) Selective reporting (repor } \\
\text { (G) Other biases }\end{array}$ & $\begin{array}{l}\text { on (sele } \\
\text { lection } \mathrm{t} \\
\text { d person } \\
\text { ment (d } \\
\text { ttrition } \\
\text { hg bias) }\end{array}$ & $\begin{array}{l}\text { tion bia } \\
\text { ias) } \\
\text { hel (perf } \\
\text { tection } \\
\text { ias) }\end{array}$ & $\begin{array}{l}\text { irmanc } \\
\text { ias) }\end{array}$ & bias) & & & & & Favo & (control) & Favours & veratrol) & \\
\hline
\end{tabular}

Figure 10: HDL-C.

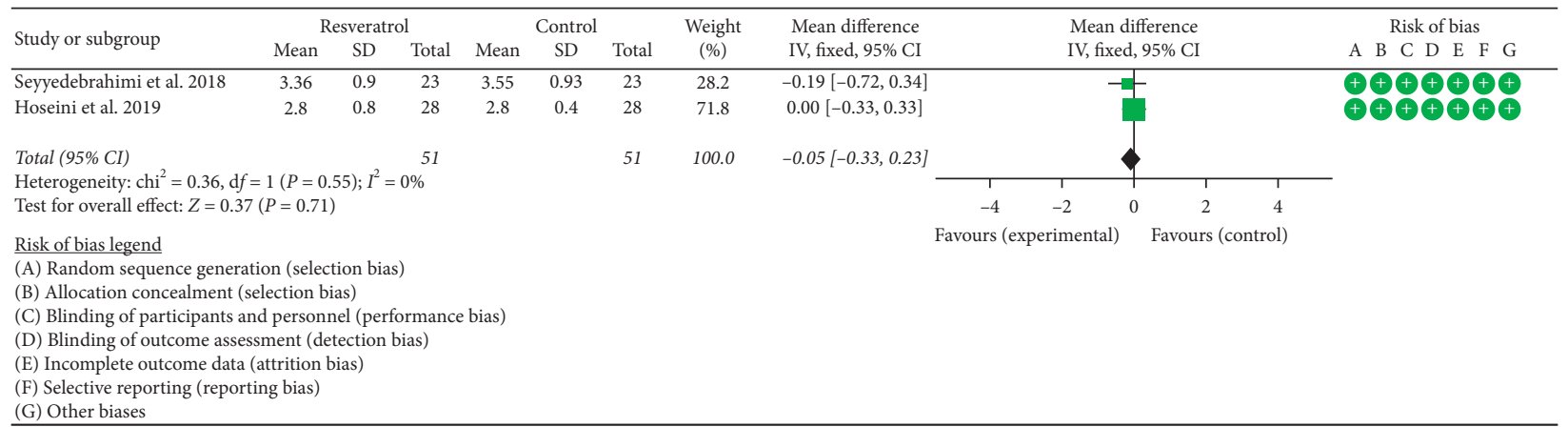

Figure 11: MDA.

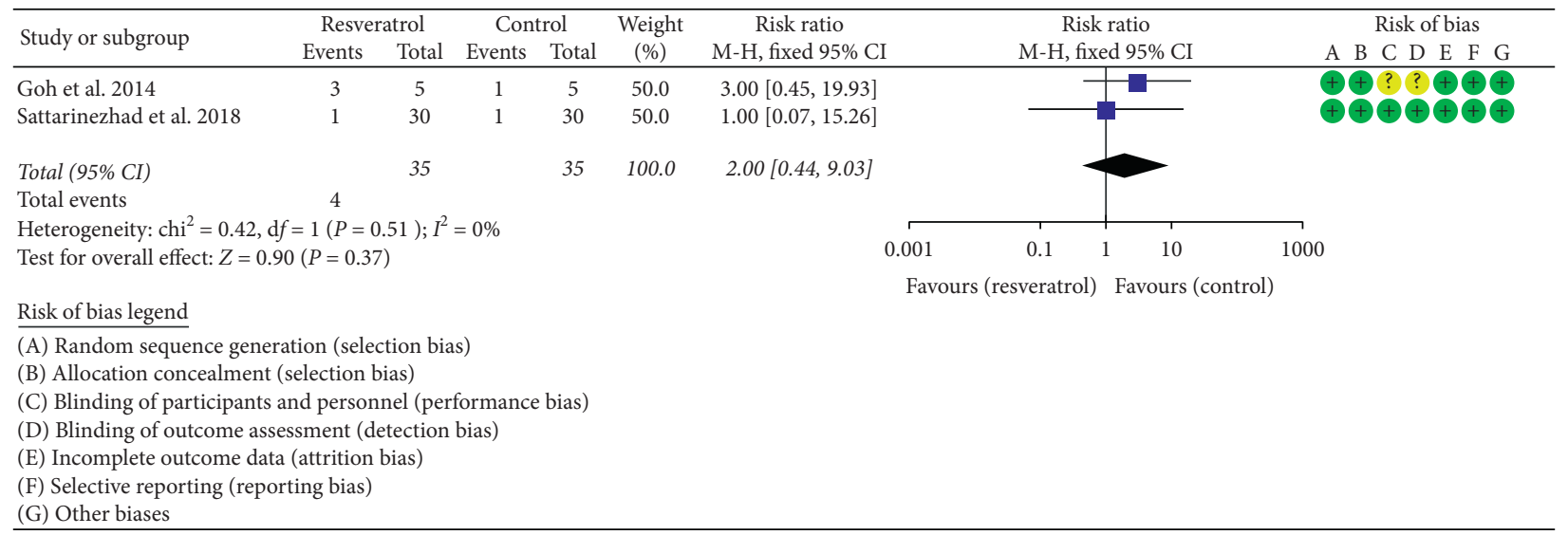

FIgURE 12: Adverse events.

Assessment. Cochrane bias risk assessment tool is used to evaluate the literature quality. (3) Included Literature. Liu et al. [14] only evaluated RCTs of types 1 and 2 diabetes, while Zhu et al. [13] only evaluated nine RCTs. This study evaluated 15 diabetes-related RCTs; 10 of them
[15-19, 29-36] are published after 2016, which showed more stable results.

The advantage of this study is that the systematic review and meta-analysis include all available RCTs for clinical problems. The study is the latest systematic review and meta- 


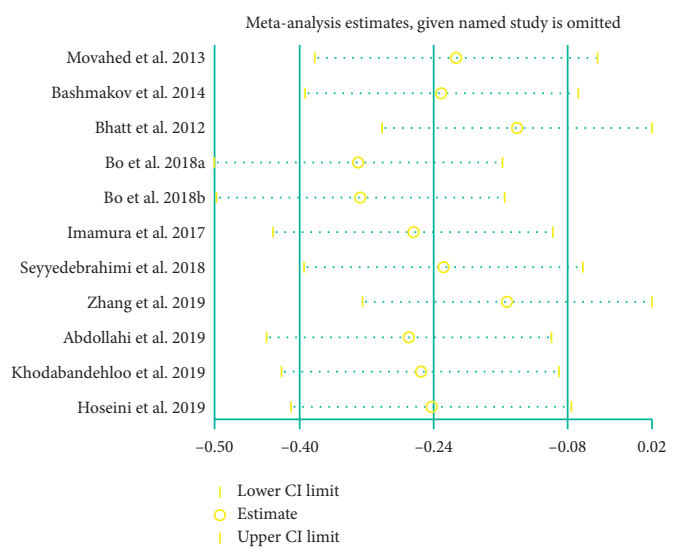

(a)

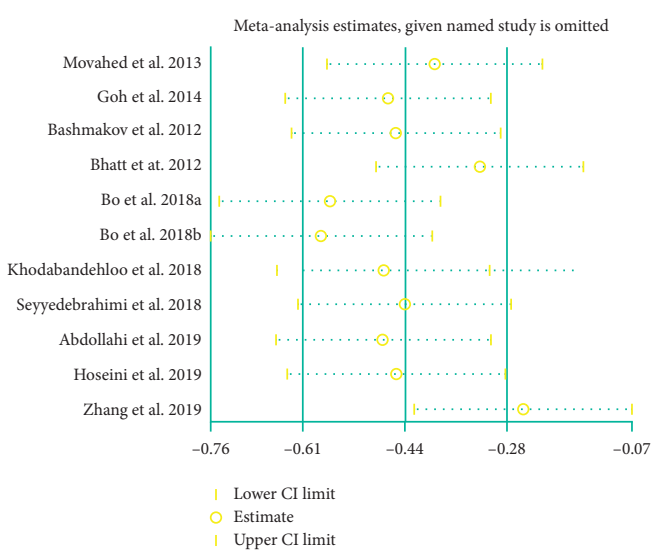

(b)

FIgURE 13: Sensitivity analysis results: (a) TC; (b) LDL-C.

analysis on this topic, and it is conducted in strict accordance with the guidelines and protocol. In addition, this study included a wider range of populations, including Iran, China, Japan, Egypt, Singapore, Hungary, and Italy, which promoted the applicability of the conclusions. The limitations of this study are as follows: (1) some RCTs are of low quality because they did not describe specific random sequence generation methods, allocation concealment methods, or blind methods, which lead to a decrease in the reliability of the results, and the results should be treated with caution in clinical practice. For example, five RCTs $[15,17,26,27,36]$ did not describe the method of generating random sequences; six RCTs $[15,17,26,27,35,36]$ did not describe the allocation concealment method; three RCTs [28, 35, 36] did not specify whether blinding; six RCTs $[17,18,25,31,32,35]$ did not describe whether to use intendto-treat analysis. (2) Some RCTs involve few participants (lower than 50), which may lead to changes in clinical efficacy indicators that cannot be detected. Goh et al. [25] included only 10 participants; Brasnyó et al. [26], 19 participants; Bashmakov et al. [27], 24 participants; Zare Javid et al. [15], 43 participants; Khodabandehloo et al. [19], 45 participants; Seyyedebrahimi et al. [31], 46 participants. (3) The different resveratrol preparations used by RCTs may affect the accuracy of the results. For example, the dose and usage of resveratrol in the study of Brasnyó et al. [26] was $5 \mathrm{mg}$, bid; in the study of Bashmakov et al. [27], $50 \mathrm{mg}$, bid; in the study of Movahed et al. [24], $500 \mathrm{mg}$, bid. The dosage of resveratrol in each RCT was different. (4) The heterogeneity of most outcomes, such as HOMA-IR, TC, TG, and LDL-C, was high. The high heterogeneity may reduce the applicability of the results. Therefore, high-quality research is needed to determine or modify the results of this research.

\section{Conclusion}

Resveratrol may improve insulin resistance, lower fasting blood glucose and insulin levels, and improve oxidative stress in patients with T2DM. However, due to the generally low quality of research and high heterogeneity among RCTs, the results should be interpreted with caution.

\section{Data Availability}

All data generated or analyzed during this study are included within the article.

\section{Disclosure}

Tianqing Zhang and Qi He are the co-first authors. Hengjing $\mathrm{Hu}$ is the corresponding author.

\section{Conflicts of Interest}

The authors declare that there are no conflicts of interest.

\section{Authors' Contributions}

Tianqing Zhang, Qi He, and Hengjing Hu are responsible for the study concept and design. Tianqing Zhang, Qi He, Yao Liu, Zhenrong Chen, and Hengjing $\mathrm{Hu}$ are responsible for the literature search. Tianqing Zhang, Qi He, Yao Liu, Zhenrong Chen, and Hengjing $\mathrm{Hu}$ are responsible for data analysis and interpretation. Tianqing Zhang and Qi He drafted the paper. Hengjing $\mathrm{Hu}$ supervised the study. All authors participated in the analysis and interpretation of data and approved the final paper.

\section{Acknowledgments}

This work was supported by grants from the National Natural Science Foundation of China (grant no. 81700306), Natural Science Foundation of Hunan Province (grant no. 2018JJ3469), and China Postdoctoral Science Foundation (grant no. 2017M622588). Thanks are due to Kailin Yang for his guidance on the systematic review and meta-analysis.

\section{Supplementary Materials}

The "PRISMA 2020 checklist" is a standardized checklist that ensures that systematic reviews and meta-analyses are implemented according to this standard. Table S1: search strategies for PubMed. (Supplementary Materials) 


\section{References}

[1] P. Saeedi, I. Petersohn, P. Salpea et al., "Global and regional diabetes prevalence estimates for 2019 and projections for 2030 and 2045: results from the international diabetes federation diabetes atlas, 9th edition," Diabetes Research and Clinical Practice, vol. 157, Article ID 107843, 2019.

[2] GBD 2015 Mortality and Causes of Death Collaborators, "Global, regional, and national life expectancy, all-cause mortality, and cause-specific mortality for 249 causes of death, 1980-2015: a systematic analysis for the global burden of disease study 2015," Lancet, vol. 388, pp. 1459-1544, 2016.

[3] J. M. Forbes and M. E. Cooper, "Mechanisms of diabetic complications," Physiological Reviews, vol. 93, no. 1, pp. 137-188, 2013.

[4] M. Stolar, "Glycemic control and complications in type 2 diabetes mellitus," The American Journal of Medicine, vol. 123, pp. S3-11, 2010.

[5] D. Wu, D. Hu, H. Chen et al., "Glucose-regulated phosphorylation of TET2 by AMPK reveals a pathway linking diabetes to cancer," Nature, vol. 559, no. 7715, pp. 637-641, 2018.

[6] R. M. Carrillo-Larco, J. Pearson-Stuttard, A. Bernabe-Ortiz, and E. W. Gregg, "The andean latin-American burden of diabetes attributable to high body mass index: a comparative risk assessment," Diabetes Research and Clinical Practice, vol. 160, Article ID 107978, 2020.

[7] A. J. Vegas, O. Veiseh, M. Gürtler et al., "Long-term glycemic control using polymer-encapsulated human stem cell-derived beta cells in immune-competent mice," Nature Medicine, vol. 22, no. 3, pp. 306-311, 2016.

[8] A. Y. Y. Cheng and I. G. Fantus, "Oral antihyperglycemic therapy for type 2 diabetes mellitus," Canadian Medical Association Journal, vol. 172, no. 2, pp. 213-226, 2005.

[9] G. Nicholson and G. M. Hall, "Diabetes mellitus: new drugs for a new epidemic," British Journal of Anaesthesia, vol. 107, no. 1, pp. 65-73, 2011.

[10] E. J. Verspohl, "Novel pharmacological approaches to the treatment of type 2 diabetes," Pharmacological Reviews, vol. 64, no. 2, pp. 188-237, 2012.

[11] T. Szkudelski and K. Szkudelska, "Resveratrol and diabetes: from animal to human studies," Biochimica et Biophysica Acta (BBA)-Molecular Basis of Disease, vol. 1852, no. 6, pp. 1145-1154, 2015.

[12] E. Öztürk, A. K. K. Arslan, M. B. Yerer, and A. Bishayee, "Resveratrol and diabetes: a critical review of clinical studies," Biomedicine \& Pharmacotherapy, vol. 95, pp. 230-234, 2017.

[13] X. Zhu, C. Wu, S. Qiu, X. Yuan, and L. Li, "Effects of resveratrol on glucose control and insulin sensitivity in subjects with type 2 diabetes: systematic review and metaanalysis," Nutrition \& Metabolism, vol. 14, no. 1, p. 60, 2017.

[14] K. Liu, R. Zhou, B. Wang, and M.-T. Mi, "Effect of resveratrol on glucose control and insulin sensitivity: a meta-analysis of 11 randomized controlled trials," The American Journal of Clinical Nutrition, vol. 99, no. 6, pp. 1510-1519, 2014.

[15] A. Zare Javid, R. Hormoznejad, H. A. Yousefimanesh et al., "The impact of resveratrol supplementation on blood glucose, insulin, insulin resistance, triglyceride, and periodontal markers in type 2 diabetic patients with chronic periodontitis," Phytotherapy Research, vol. 31, no. 1, pp. 108-114, 2017.

[16] S. Bo, V. Ponzo, A. Evangelista et al., "Effects of 6 months of resveratrol versus placebo on pentraxin 3 in patients with type 2 diabetes mellitus: a double-blind randomized controlled trial," Acta Diabetologica, vol. 54, no. 5, pp. 499-507, 2017.
[17] H. Imamura, T. Yamaguchi, D. Nagayama, A. Saiki, K. Shirai, and I. Tatsuno, "Resveratrol ameliorates arterial stiffness assessed by cardio-ankle vascular index in patients with type 2 diabetes mellitus," International Heart Journal, vol. 58, no. 4, pp. 577-583, 2017.

[18] A. Sattarinezhad, J. Roozbeh, B. Shirazi Yeganeh, G. R. Omrani, and M. Shams, "Resveratrol reduces albuminuria in diabetic nephropathy: a randomized double-blind placebo-controlled clinical trial," Diabetes \& Metabolism, vol. 45, no. 1, pp. 53-59, 2019.

[19] H. Khodabandehloo, S. Seyyedebrahimi, E. N. Esfahani, F. Razi, and R. Meshkani, "Resveratrol supplementation decreases blood glucose without changing the circulating CD $14^{+}$ $\mathrm{CD}_{16}{ }^{+}$monocytes and inflammatory cytokines in patients with type 2 diabetes: a randomized, double-blind, placebocontrolled study," Nutrition Research, vol. 54, pp. 40-51, 2018.

[20] M. de Ligt, Y. M. H. Bruls, J. Hansen et al., "Resveratrol improves ex vivo mitochondrial function but does not affect insulin sensitivity or brown adipose tissue in first degree relatives of patients with type 2 diabetes," Molecular Metabolism, vol. 12, pp. 39-47, 2018.

[21] J. J. Deeks, J. P. Higgins, and D. G. Altman, "Chapter 16: special topics in statistics," in Cochrane Handbook for Systematic Reviews of Interventions, J. P. Higgins and S. Green, Eds., The Cochrane Collaboration, London, UK, 2020.

[22] J. J. Deeks, J. P. Higgins, and D. G. Altman, Edited by J. P. Higgins, Ed., "Chapter 8: assessing risk of bias in included studies," in Cochrane Handbook or Systematic Reviews of Interventions Version 6.1.0, S. Green, Ed., The Cochrane Collaboration, London, UK, 2020.

[23] J. J. Deeks, J. P. Higgins, and D. G. Altman, "Chapter 9: analyzing data and undertaking meta-analyses," in Cochrane Handbook for Systematic Reviews of Interventions, J. P. Higgins and S. Green, Eds., The Cochrane Collaboration, London, UK, 2020.

[24] A. Movahed, I. Nabipour, X. Lieben Louis et al., "Antihyperglycemic effects of short term resveratrol supplementation in type 2 diabetic patients," Evidence-based Complementary and Alternative Medicine: ECAM, vol. 2013, Article ID 851267, 11 pages, 2013.

[25] K. P. Goh, H. Y. Lee, D. P. Lau, W. Supaat, Y. H. Chan, and A. F. Y. Koh, "Effects of resveratrol in patients with type 2 diabetes mellitus on skeletal muscle SIRT1 expression and energy expenditure," International Journal of Sport Nutrition and Exercise Metabolism, vol. 24, no. 1, pp. 2-13, 2014.

[26] P. Brasnyó, G. A. Molnár, M. Mohás et al., "Resveratrol improves insulin sensitivity, reduces oxidative stress and activates the Akt pathway in type 2 diabetic patients," British Journal of Nutrition, vol. 106, no. 3, pp. 383-389, 2011.

[27] Y. K. Bashmakov, S. H. Assaad-Khalil, M. Abou Seif et al., "Resveratrol promotes foot ulcer size reduction in type 2 diabetes patients," ISRN Endocrinology, vol. 2014, Article ID 816307, 8 pages, 2014.

[28] J. K. Bhatt, S. Thomas, and M. J. Nanjan, "Resveratrol supplementation improves glycemic control in type 2 diabetes mellitus," Nutrition Research, vol. 32, no. 7, pp. 537-541, 2012.

[29] S. Bo, V. Ponzo, G. Ciccone et al., "Six months of resveratrol supplementation has no measurable effect in type 2 diabetic patients. a randomized, double blind, placebo-controlled trial," Pharmacological Research, vol. 111, pp. 896-905, 2016.

[30] S. Bo, R. Gambino, V. Ponzo et al., "Effects of resveratrol on bone health in type 2 diabetic patients. a double-blind randomizedcontrolled trial," Nutrition \& Diabetes, vol. 8, no. 1, p. 51, 2018. 
[31] S. Seyyedebrahimi, H. Khodabandehloo, E. Nasli Esfahani, and R. Meshkani, "The effects of resveratrol on markers of oxidative stress in patients with type 2 diabetes: a randomized, double-blind, placebo-controlled clinical trial," Acta Diabetologica, vol. 55, no. 4, pp. 341-353, 2018.

[32] A. Hoseini, G. Namazi, A. Farrokhian et al., "The effects of resveratrol on metabolic status in patients with type 2 diabetes mellitus and coronary heart disease," Food \& Function, vol. 10, no. 9, pp. 6042-6051, 2019.

[33] S. Abdollahi, A. Salehi-Abargouei, O. Toupchian et al., "The effect of resveratrol supplementation on cardio-metabolic risk factors in patients with type 2 diabetes: a randomized, doubleblind controlled trial," Phytotherapy Research, vol. 33, no. 12, pp. 3153-3162, 2019.

[34] M. Tabatabaie, S. Abdollahi, A. Salehi-Abargouei et al., "The effect of resveratrol supplementation on serum levels of asymmetric de-methyl-arginine and paraoxonase 1 activity in patients with type 2 diabetes: a randomized, double-blind controlled trial," Phytotherapy Research, vol. 34, no. 8, pp. 2023-2031, 2020.

[35] H. Zhang, A. Li, H. Xiao, D. Wang, and L. Zhang, "Effects of resveratrol on blood glucose, blood lipids and hemorheology in patients with type 2 diabetes," Modern Journal of Integrated Traditional Chinese and Western Medicine, vol. 28, no. 17, pp. 1888-1891, 2019.

[36] J. Ying, J. Li, and S. Yu, "The value of resveratrol soft capsules in the treatment of early retinopathy and its effect on blood glucose in diabetic patients," China Modern Doctor, vol. 55, no. 6, pp. 4-7, 2017.

[37] T. H. Sanders, R. W. Mc Michael Jr, and K. W. Hendrix, "Occurrence of resveratrol in edible peanuts," Journal of Agricultural and Food Chemistry, vol. 48, no. 4, pp. 1243-1246, 2000.

[38] M. Shakibaei, K. B. Harikumar, and B. B. Aggarwal, "Resveratrol addiction: to die or not to die," Molecular Nutrition \& Food Research, vol. 53, no. 1, pp. 115-128, 2009.

[39] M.-E. García-Pérez, M. Royer, G. Herbette, Y. Desjardins, R. Pouliot, and T. Stevanovic, "Picea mariana bark: a new source of trans-resveratrol and other bioactive polyphenols," Food Chemistry, vol. 135, no. 3, pp. 1173-1182, 2012.

[40] L. Nemcova, J. Zima, J. Barek, and D. Janovská, "Detemination of resveratrol in grains, hulls and leaves of common and tartary buckwheat by HPLC with electrochemical detection at carbon paste electrode," Food Chemistry, vol. 126, no. 1, pp. 374-378, 2011.

[41] A. Betelli, A. A. Bertelli, A. Gozzini, and L. Giovannini, "Plasma and tisue resveratrol concentrations and pharmacological activity," Drugs Under Experimental and Dinical Research, vol. 24, no. 3, pp. 133-138, 1998.

[42] S. Fogacci, F. Fogacci, and A. F. G. Cicero, "Nutraceuticals and hypertensive disorders in pregnancy: the available clinical evidence," Nutrients, vol. 12, no. 2, p. 378, 2020.

[43] C. Borghi, K. Tsioufis, E. Agabiti-Rosei et al., "Nutraceuticals and blood pressure control: a European society of hypertension position document," Journal of Hypertension, vol. 38, no. 5, pp. 799-812, 2020.

[44] F. Fogacci, M. Banach, and A. F. G. Cicero, "Resveratrol effect on patients with non-alcoholic fatty liver disease: a matter of dose and treatment length," Diabetes, Obesity and Metabolism, vol. 20, no. 7, pp. 1798-1799, 2018.

[45] C. R. Sirtori, A. Arnoldi, and A. F. G. Cicero, "Nutraceuticals for blood pressure control," Annals of Medicine, vol. 47, no. 6, pp. 447-456, 2015.
[46] M. Sadria and A. T. Layton, "Aging affects circadian clock and metabolism and modulates timing of medication," iScience, vol. 24, no. 4, Article ID 102245, 2021.

[47] M. D. van Die, S. G. Williams, J. Emery et al., "A placebocontrolled double-blinded randomized pilot study of combination phytotherapy in biochemically recurrent prostate cancer," The Prostate, vol. 77, no. 7, pp. 765-775, 2017.

[48] J. L. Espinoza, L. Q. Trung, P. T. Inaoka et al., "The repeated administration of resveratrol has measurable effects on circulating T-cell subsets in humans," Oxidative Medicine and Cellular Longevity, vol. 2017, Article ID 6781872, 10 pages, 2017.

[49] L. D. Williams, G. A. Burdock, J. A. Edwards, M. Beck, and J. Bausch, "Safety studies conducted on high-purity transresveratrol in experimental animals," Food and Chemical Toxicology, vol. 47, no. 9, pp. 2170-2182, 2009.

[50] V. Hebbar, G. Shen, R. Hu et al., "Toxicogenomics of resveratrol in rat liver," Life Sciences, vol. 76, no. 20, pp. 2299-2314, 2005.

[51] W. D. Johnson, R. L. Morrissey, A. L. Usborne et al., "Subchronic oral toxicity and cardiovascular safety pharmacology studies of resveratrol, a naturally occurring polyphenol with cancer preventive activity," Food and Chemical Toxicology, vol. 49, no. 12, pp. 3319-3327, 2011.

[52] K. Kantartzis, L. Fritsche, M. Bombrich et al., "Effects of resveratrol supplementation on liver fat content in overweight and insulin-resistant subjects: a randomized, double-blind, placebo-controlled clinical trial," Diabetes, Obesity and Metabolism, vol. 20, no. 7, pp. 1793-1797, 2018.

[53] F. Fogacci, G. Tocci, V. Presta, A. Fratter, C. Borghi, and A. F. G. Cicero, "Effect of resveratrol on blood pressure: a systematic review and meta-analysis of randomized, controlled, clinical trials," Critical Reviews in Food Science and Nutrition, vol. 59, no. 10, pp. 1605-1618, 2019. 